\title{
Control Constraints, Abnormality, and Improved Performance by Periodic Control
}

\author{
DENNIS S. BERNSTEIN, MEMBER, IEEE
}

\begin{abstract}
Second-order conditions for steady-state optimality and nonoptimality in a periodic control problem are presented. The main result is a generalization of the $\Pi$ test, a second-order sufficient condition for improved performance by periodic control. Earlier results are generalized in two distinct ways: 1) the control constraint set is only assumed to be convex (and, hence, possibly nonopen) thus allowing the optimal steady-state control to be an element of the control constraint set boundary; and 2) auxiliary normality conditions are eliminated. Proofs of the results are based upon second-order necessary conditions for nonlinear programming and optimal control obtained recently in [43] and [44].
\end{abstract}

\section{INTRODUCTION}

$\mathbf{F}$

OR the autonomous infinite-interval control process

$$
\dot{x}(t)=f(x(t), u(t)), \quad t \in[0, \infty)
$$

one may utilize either of the cost functionals

$$
J=\int_{0}^{\infty} \tilde{f}(x(t), u(t)) d t
$$

or

$$
J=\lim _{\tau \rightarrow \infty} \frac{1}{\tau} \int_{0}^{\tau} f(x(t), u(t)) d t
$$

depending upon physical or mathematical considerations. After dissipation of transients due to initial conditions or disturbances, control strategies should be capable of indefinite application, the simplest operation being steady-state, i.e.,

$$
0=f(\bar{x}, \bar{u}),
$$

where $\bar{x}$ and $\bar{u}$ are constant. The approach of periodic control is to consider controls and states that satisfy a periodicity condition

$$
x(0)=x(\tau)
$$

with the goal of minimizing an average criterion

$$
J=\frac{1}{\tau} \int_{0}^{\tau} \tilde{f}(x(t), u(t)) d t
$$

The reasoning behind (1.1) and (1.2) is that any control which is optimal over $[0, \tau]$ can be repeated in $[\tau, 2 \tau],[2 \tau, 3 \tau]$, and so forth. The central questions of periodic control are: 1) when does time-dependent (periodic) control produce better performance than constant (steady-state) control (this situation is called

Manuscript received March 11, 1983; revised January 6, 1984. Paper recommended by Past Associate Editor, B. R. Barmish. This work was supported in part by the Air Force Office of Scientific Research under Grant AFOSR-77-3158.

The author is with the Controls Analysis and Synthesis Group, Government Aerospace Systems Division, Harris Corporation, Melbourne, FL 32901. “proper"'); and 2) what mechanisms are responsible for effecting this improvement.

Most of the applications of periodic control have been in two areas, namely, chemical process control and aircraft performance optimization. Continuous stirred tank reactors provided the earliest motivation for "unsteady-state" processing by the presence of situations in which periodic control could increase product selectivity or yield [1]-[5]. Periodic operation of aircraft has focused on the problems of maximum range, maximum endurance, and peak altitude [6]-[14]. In particular, the nonoptimality of steady-state cruise has been a much-debated issue [6]-[10]. Periodic operation has also been considered for problems in unpowered flight, specifically, the soaring of gliders and birds [15], [16]. Most surprising is the possibility of sustained unpowered flight in wind possessing a horizontal velocity gradient but no vertical velocity component. Other application areas for periodic control include solar energy collection [17], economic theory [18], and cardio-circulatory assist devices [19].

Theoretical approaches to periodic control usually involve optimality conditions as tests for proper. Since the periodic control problem is a specialized optimal control problem with side constraints and boundary conditions, much of the theory mirrors results from the optimal control literature. Interesting issues arise because the periodic control problem has embedded within it a relatively simpler steady-state problem. First-order conditions to determine proper [20]-[22] are based upon strong control variations, and hence are similar to the maximum principle. Weak control variations, on the other hand, are useless in ascertaining proper in a first-variation analysis since the average value of their dynamic components (i.e., nonzero-frequency harmonics) is zero.

A second-ơrder sufficient condition for proper based upon weak control variations was first given in [23] and was subsequently generalized to problems involving side constraints in [24]. This condition, called the $\Pi$ test, consists of verifying that a quadratic form [involving a frequency-dependent matrix $\Pi(\omega)$ ] satisfies a sign condition. It was subsequently shown in [25] and [26] that the $\Pi$ test of [24] may incorrectly predict proper unless a normality condition holds. Also, in [23]-[26] it is assumed that the optimal steady-state control $\bar{u}$ lies in the interior of the control constraint set. This, of course, simplifies matters since local control variations can be arbitrarily selected. In certain applications, however, $\bar{u}$ lies in the boundary of the control constraint set. For example, in the presence of an altitude constraint for the maximum-range cruise problem, the optimal steady-state cruise path can occur at maximum thrust [13], [14]. The purpose of the present paper, then, is to extend the $\Pi$ test in two distinct ways: to eliminate the normality condition; and to allow the optimal steadystate control to lie in the boundary of a (nonopen) convex control constraint set. ${ }^{1}$

Although first-order optimality conditions such as the maxi-

${ }^{1}$ It should be noted that a II test was derived in [27] for a problem in which the control constraint set is of the form $\left\{u \in \mathbb{R}^{m}: w(u) \geq 0\right\}$. Since this assumption and the convex control constraint set assumption of the present paper are generally different, it is difficult to compare directly our results to those of [27]. 
mum principle allow for general control constraint sets, classical second-order necessary conditions assume that the control constraint set is open (see, e.g., [28]). Second-order necessary conditions for optimal control with a (nonopen) convex control constraint set were first derived in [29]-[31], where weak, strong, and "hybrid" variations were treated, respectively. Although it would indeed be possible to derive a $\Pi$ test with convex control constraint set directly from the second-order necessary conditions of [29], this would achieve only half the goal of the present paper since the results of [29] involve a normality assumption as in [25] and [26].

Regularity assumptions such as normality conditions in optimal control [28]-[33] and constraint qualifications in nonlinear programming [34] are persistent features of optimality theory. Although such conditions often imply the existence of a unique Lagrange multiplier with nonzero component corresponding to the cost functional, they are usually stronger than necessary and unpleasant to verify. Recently, higher order necessary conditions for optimization [35]-[43] and optimal control [44] have been developed which do not require an auxiliary normality condition. ${ }^{2}$ The idea behind these results is to define a set of "critical directions" which, to a first-order approximation, have inferior cost and satisfy the problem constraints. The key feature is that because of the lack of a normality condition, the multiplier satisfying the second-order necessary conditions depends on the choice of critical direction. This directly accounts for the nonuniqueness of the multiplier and clarifies the role of normality conditions when they hold, i.e., to imply the existence of a unique multiplier.

The paper is organized as follows. In Section II the optimal periodic control problem (OPC) and optimal steady-state problem (OSS) are stated along with some preliminary definitions and results for use in later sections. First- and second-order necessary conditions for OSS are given in Section III. In Section IV we present first- and second-order necessary conditions for steadystate optimality in OPC in a general form, i.e., for state and control variations given by Fourier expansions. The generalized $\Pi$ test is given in Section $V$ for sinusoidal control variations. Section VI presents some illustrative examples and brief concluding remarks are given in Section VII. Appendix A presents technical lemmas concerning periodic solutions of linear systems and Appendix B contains necessary conditions for optimal control based upon results obtained in [44]. Finally, the proof of Theorem 4.1 , the key result in Section IV, is given in Appendix C.

\section{General Notation and Problem Formulation}

Let $R, G$, and $N$ denote, respectively, the real field, complex field, and set of positive integers. For $w \hat{=}\left(w_{1}, \cdots, w_{s}\right) \in \mathcal{B}^{s}$, $|w| \triangleq \sum_{i=1}^{s}\left|w_{i}\right|$. If $I \subset \mathbb{R}$ is an interval and $W \subset \mathbb{R}^{s}$, then $A C(I$, $W), M(I, W)$ and $L_{p}(I, W)(p \in[1, \infty])$ denote, respectively, the set of functions $h: I \rightarrow W$ which are absolutely continuous, measurable, and $L_{p}$-integrable or essentially bounded, i.e., the norm

$$
\begin{aligned}
\|h\|_{p, I} & \triangleq\left[\int_{I}|h(t)|^{p} d t\right]^{1 / p}, \quad p \in[1, \infty) \\
& \triangleq \operatorname{ess} \sup _{t \in I}|h(t)|, \quad p=\infty
\end{aligned}
$$

is finite. Let $j \triangleq \sqrt{-1}$ and let $I_{s}$ denote the $s \times s$ identity matrix. If $w$ is a scalar, vector or matrix with elements in $\bar{C}$, then $\bar{w}, w^{T}$, $w^{*}, \operatorname{Re} w$, and $\operatorname{Im} w$ denote, respectively, the complex conjugate, transpose, conjugate transpose, real part, and imaginary part of $w$. If $W \subset\left[\mathrm{B}^{s}\right.$, then int $W$ is the interior of $W$ and cone $\mathrm{W} \triangleq$ $\{\alpha w: \alpha>0, w \in W\}$. Throughout this paper, $\tau$ is a positive number

\footnotetext{
${ }^{2}$ Actually, in $1942 \mathrm{McShane}$ [45] stated second-order necessary conditions for a nonlinear programming problem without a normality condition.
}

To state the optimal periodic control problem we require the following notation. Let $n, m, l \in \mathbb{N}, j, k \in\{0\} \cup \mathrm{N}, X \subset \mathbb{R}^{n}$, $U \subset \mathbb{R}^{m}$, and $Y \subset \mathbb{R}^{l}$ be open, $U_{c} \subset U$ be convex, $g_{i}: Y \rightarrow$ 思, $i$ $\in\{0, \cdots, j\}, h: Y \rightarrow \mathrm{R}^{k}$ (omit if $k=0$ ), $f: X \times U \rightarrow \mathbb{R}^{n}$, and $f: X \times U \rightarrow\left[\mathbb{R}^{\prime}\right.$. Also define $\mathfrak{X}_{\tau} \triangleq A C([0, \tau], X)$ and $\mathfrak{u}_{\tau} \triangleq$ $M\left([0, \tau], U_{c}\right)$.

\section{Optimal Period Control Problem (OPC)}

Find $\tau$ and $(x(\cdot), u(\cdot)) \in \mathfrak{X}_{\tau} \times \mathcal{u}_{\tau}$ which minimize

$$
J_{P C}(x(\cdot), u(\cdot), \tau) \triangleq g_{0}(y)
$$

subject to

$$
\begin{gathered}
g_{i}(y) \leq 0, i \in\{1, \cdots, j\} \text { (omit if } j=0 \text { ), } \\
h(y)=0 \text { (omit if } k=0), \\
y=\frac{1}{\tau} \int_{0}^{\tau} \tilde{f}(x(t), u(t)) d t \in Y, \\
\dot{x}(t)=f(x(t), u(t)), \text { a.a. } t \in[0, \tau], \\
x(0)=x(\tau) .
\end{gathered}
$$

When the period $\tau$ is fixed in OPC (and, hence, the optimization is only over $\mathfrak{X}_{\tau} \times \mathcal{U}_{\tau}$ ), we denote the problem by OPC $(\tau)$.

\section{Optimal Steady-State Problem (OSS)}

Find $(x, u) \in X \times U_{c}$ which minimizes

$$
J_{S S}(x, u) \triangleq g_{0}(y)
$$

subject to $(2.2),(2.3)$,

$$
\begin{gathered}
y=\tilde{f}(x, u) \in Y, \\
f(x, u)=0 .
\end{gathered}
$$

We next consider some terminology for these problems. The triple $(x(\cdot), u(\cdot), \tau)$, where $(x(\cdot), u(\cdot)) \in \mathscr{X}_{\tau} \times u_{\tau}$, is admissible if (2.2)-(2.6) are satisfied; the pair $(x, u) \in X \times U_{c}$ is steady-state admissible if (2.2), (2.3), (2.8), and (2.9) are satisfied. A steady-state admissible pair $(\bar{x}, \bar{u})$ is a local minimum of OSS if there exists $\epsilon>0$ such that for all steady-state admissible pairs $(x, u)$ satisfying $|x-\bar{x}|+|u-\bar{u}|<\epsilon$ it follows that $J_{S S}(\bar{x}, \bar{u}) \leq J_{S S}(x, u)$. A local minimum $(\bar{x}, \bar{u})$ of OSF is a local steady-state minimum of OPC if there exists $\epsilon>$ 0 such that for all admissible triples $(x(\cdot), u(\cdot), \tau)$ satisfying $\| x(\cdot)$ $-\bar{x}\left\|_{\infty,[0, \tau]}+\right\| u(\cdot)-\bar{u} \|_{\infty,[0, \tau]}<\epsilon$ it follows that $J_{S S}(\bar{x}, \bar{u}) \leq$ $J_{P C}(x(\cdot), u(\cdot), \tau)$. If a local minimum $(\bar{x}, \bar{u})$ of OSS is not a local steady-state minimum of OPC, then OPC is locally proper at $(\bar{x}$, $\bar{u})$. Thus, OPC is locally proper at $(\bar{x}, \bar{u})$ if and only if for all $\epsilon>0$ there exists an admissible triple $(x(\cdot), u(\cdot), \tau)$ such that $\| x(\cdot)-$ $\bar{x}\left\|_{\infty,\{0, \tau]}+\right\| u(\cdot)-\bar{u} \|_{\infty,[0, \tau]}<\epsilon$ and $J_{P C}(x(\cdot), u(\cdot), \tau)<J_{S S}(\bar{x}$ $\vec{u})$. These definitions will also be used when $\mathrm{OPC}$ is replaced by $\operatorname{OPC}(\tau)$, i.e., when $\tau$ is fixed. Note that if $\mathrm{OPC}(\tau)$ is locally proper, then improving controls can be found with period $\tau$.

\section{NECESSARY CONDITIONS FOR OPTIMALITY IN OSS}

In this section we present Theorem 3.1, the first- and secondorder necessary conditions for a local minimum of OSS. Since OSS is a finite-dimensional optimization problem with equality, inequality, and set constraints, necessary conditions can be obtained by applying known results from nonlinear programming. In particular, [43, Theorem 6.1] can be used since it possesses sufficient generality for our purposes. The derivation of Theorem 3.1 will be omitted, however, since it is straightforward. We also state a specialized version of Theorem 3.1, Corollary 3.1 , for the 
case det $A \neq 0$. Simplification is obtained in this case since state variations are uniquely determined by control variations. The remainder of the section concerns normality conditions for determining the existence of a unique multiplier satisfying the first-order necessary conditions.

Before proceeding we shall invoke two assumptions which will simplify the notation in this and the following two sections. First, we assume that all inequality constraints are active at the optimal pair $(\bar{x}, \bar{u})$, i.e., if $j>0$, then $g_{i}(\bar{y})=0, i=1, \cdots, j$, where $\bar{y}$ $\triangleq \tilde{f} \bar{x}, \bar{u})$. There is no loss of generality in this assumption since we are concerned with local optimality. And second, statements of the results are confined to the case $k>0$. Specialization to the case $k=0$ is obvious.

The notation for derivatives and partial derivatives is standard. We only note that if $\gamma \triangleq\left(\gamma_{1}, \cdots, \gamma_{b}\right): E \subset \mathbb{R}^{a} \rightarrow \mathbb{R}^{b}$, where $E$ is open and $\bar{e} \in E$, then $\gamma^{\prime}(\bar{e}): \mathbb{R}^{a} \rightarrow \mathbb{R}^{b}$ denotes the first (Frechet) derivative (Jacobian matrix) of $\gamma$ at $\bar{e}$ and $\gamma^{\prime \prime}(\bar{e}): \mathbb{R}^{a} \times \mathbb{R}^{a} \rightarrow \mathbb{R}^{b}$, the second (Frechet) derivative of $\gamma$ at $\bar{e}$, is the bilinear map identified with the Hessian matrices of the components of $\gamma$. Specifically, the $i$ th component of $\eta_{1}^{T} \gamma^{\prime \prime}(\bar{e}) \eta_{2}$ is $\eta_{1}^{T} \gamma_{i}^{\prime \prime}(\bar{e}) \eta_{2}$, where $\gamma_{i}^{\prime \prime}(\bar{e}) \in \mathbb{R}^{a \times a}$ is the matrix of second partials of $\gamma_{i}$ at $\ddot{e}$.

The necessary conditions involve a Lagrangian function $L: X \times$ $U \times Y \times \mathbb{R}^{1+j+k} \times \mathbb{R}^{n} \times \mathbb{B}^{l} \rightarrow \mathbb{R}$ defined by

$$
\begin{aligned}
L(x, u, y, \alpha, \lambda, \mu) \triangleq & \alpha^{T} g(y)+\lambda^{T} f(x, u) \\
& +\mu^{T}(\tilde{f}(x, u)-y),
\end{aligned}
$$

where

$$
\begin{gathered}
g(y) \triangleq\left(g_{0}(y), \cdots, g_{j}(y), h(y)^{T}\right)^{T}, \\
\alpha \triangleq\left(\alpha_{0}, \cdots, \alpha_{j}, \alpha_{h}^{T}\right)^{T}, \quad \alpha_{h} \in \mathbb{B}^{k} .
\end{gathered}
$$

Since several functions will be evaluted at $\bar{x}, \bar{u}$, and $\bar{y}$, we employ a super-bar notation as in $\bar{f} \triangleq f(\bar{x}, \bar{u})$ and $\bar{g}_{i y} \triangleq g_{i y}(\bar{y})$. Also, let

$$
A \triangleq \bar{f}_{x}, B \triangleq \bar{f}_{u}, C \triangleq \bar{f}_{x}, D \triangleq \overline{\tilde{f}}_{u}, M \triangleq \bar{g}_{y} .
$$

The second-order necessary conditions are stated in terms of variations in the state and control variables. Up to a first-order approximation these "critical directions" have inferior cost and satisfy the problem constraints. Thus, define

$$
D \triangleq\left\{\left(\zeta_{0}, \nu_{0}\right) \in \mathbb{R}^{n} \times\left(U_{c}-\bar{u}\right):(3.1)-(3.3) \text { are satisfied }\right\},
$$

where

$$
\begin{gathered}
\bar{g}_{i y}\left(C \zeta_{0}+D \nu_{0}\right) \leq 0, i \in\{0, \cdots, j\}, \\
\bar{h}_{y}\left(C \zeta_{0}+D \nu_{0}\right)=0, \\
A \zeta_{0}+B \nu_{0}=0 .
\end{gathered}
$$

Henceforth, we assume that $g, f$, and $\tilde{f}$ are $C^{1}$ or $C^{2}$ depending upon whether first- or second-order conditions are being considered.

Theorem 3.1: If $(\bar{x}, \vec{u})$ is a local minimum of OSS, then there exists nonzero $(\alpha, \lambda)$ satisfying

$$
\begin{gathered}
\alpha_{i} \geq 0, \quad i \in\{0, \cdots, j\}, \\
\mu=M^{T} \alpha, \\
\bar{L}_{x}(\alpha, \lambda)=0, \\
\bar{L}_{u}(\alpha, \lambda) \nu \geq 0, \quad \nu \in U_{c}-\bar{u} .
\end{gathered}
$$

Furthermore, for each $\left(\zeta_{0}, \nu_{0}\right) \in \mathbb{D}$ there exists nonzero $(\alpha, \lambda)$ satisfying (3.4)-(3.7) and

$$
\begin{aligned}
& \zeta_{0}^{T} \bar{L}_{x x}(\alpha, \lambda) \zeta_{0}+2 \zeta_{0}^{T} \bar{L}_{x u}(\alpha, \lambda) \nu_{0} \\
& \quad+\nu_{0}^{T} \bar{L}_{u u}(\alpha, \lambda) \nu_{0}+\left(C \zeta_{0}+D \nu_{0}\right)^{T} \bar{L}_{y y}(\alpha)\left(C \zeta_{0}+D \nu_{0}\right) \geq 0
\end{aligned}
$$

Note that $\mu$ does not explicitly appear in (3.6) and (3.7) because of (3.5) and that $\bar{L}_{y y}(\alpha)$ is independent of $\lambda$.

To conveniently state the specialization of Theorem 3.1 to the case in which det $A \neq 0$, define $G(\omega) \in \mathrm{C}^{n \times m}$ by

$$
G(\omega) \triangleq\left(j \omega I_{n}-A\right)^{-1} B
$$

when the indicated inverse exists. If $A$ is nonsingular, the set $D$ can clearly be replaced by

$$
\mathfrak{D}^{+} \triangleq\left\{\nu_{0} \in U_{c}-\bar{u}:(3.1)^{\prime} \text { and }(3.2)^{\prime} \text { are satisfied }\right\} \text {, }
$$

where

$$
\begin{gathered}
\bar{g}_{i y}(C G(0)+D) \nu_{0} \leq 0, \quad i \in\{0, \cdots, j\}, \\
\bar{h}_{y}(C G(0)+D) \nu_{0}=0 .
\end{gathered}
$$

Since (3.6) is equivalent to

$$
\lambda=-\left(M C A^{-1}\right)^{T} \alpha,
$$

Theorem 3.1 can now be stated entirely in terms of $\alpha$.

Corollary 3.l: If $(\bar{x}, \bar{u})$ is a local minimum of OSS and det $A$ $\neq 0$ then there exists nonzero $\alpha$ satisfying (3.4), (3.5), (3.9) and

$$
\bar{L}_{u}(\alpha) \nu \geq 0, \quad \nu \in U_{c}-\bar{u} .
$$

Furthermore, for each $\nu_{0} \in \mathcal{D}^{+}$there exists nonzero $\alpha$ satisfying (3.4), (3.5), (3.9), (3.10) and

$$
\begin{aligned}
& \nu_{0}^{T}\left[G(0)^{T} \bar{L}_{x x}(\alpha) G(0)+2 G(0){ }^{T} \bar{L}_{x u}(\alpha)\right. \\
& \left.\quad+\bar{L}_{u u}(\alpha)+(C G(0)+D)^{T} \bar{L}_{y y}(\alpha)(C G(0)+D)\right] \nu_{0} \geq 0 .
\end{aligned}
$$

Considerable simplification in the statement of Theorem 3.1 can be obtained by defining the set of normalized multipliers

$$
\mathfrak{K} \triangleq\{(\alpha, \lambda):(3.4)-(3.7) \text { are satisfied and }|(\alpha, \lambda)|=1\} .
$$

This normalization represents no loss of generality since the necessary conditions are positively homogeneous in $(\alpha, \lambda)$. Since (3.4)-(3.7) determine a (closed) dual cone, it is readily seen that $\mathfrak{N}$ is compact. The following result is equivalent to Theorem 3.1.

Corollary 3.2: If $(\bar{x}, \bar{x})$ is a local minimum of OSS, then

$$
\mathfrak{K} \neq 0 \text {. }
$$

Furthermore,

$$
\begin{aligned}
& \max _{(\alpha, \lambda) \in \mathbb{T}}\left\{\zeta_{0}^{T} \bar{L}_{x x}(\alpha, \lambda) \zeta_{0}+2 \zeta_{0}^{T} \bar{L}_{x u}(\alpha, \lambda) \nu_{0}+\nu_{0}^{T} \bar{L}_{u u}(\alpha, \lambda) \nu_{0}\right. \\
& \left.+\left(C \zeta_{0}+D \nu_{0}\right)^{T} \bar{L}_{y y}(\alpha)\left(C \zeta_{0}+D \nu_{0}\right)\right\} \geq 0, \quad\left(\zeta_{0}, \nu_{0}\right) \in D
\end{aligned}
$$

For the case det $A \neq 0$ define

$$
\begin{gathered}
\mathfrak{K}^{+} \hat{\Leftrightarrow}\{\alpha:(3.4),(3.5),(3.9) \text { and }(3.10) \text { are satisfied } \\
\text { and }|\alpha|=1\} .
\end{gathered}
$$

Corollary 3.3: If $(\bar{x}, \bar{u})$ is a local minimum of OSS and $\operatorname{det} A$ $\neq 0$, then

$$
\mathfrak{M}^{+} \neq \emptyset
$$

Furthermore,

$$
\begin{aligned}
\max _{\alpha \in \mathfrak{T}^{+}} & \left\{\nu _ { 0 } ^ { T } \left[G(0)^{T} \bar{L}_{x x}(\alpha) G(0)+2 G(0)^{T} \tilde{L}_{x u}(\alpha)\right.\right. \\
+ & \left.\left.\bar{L}_{u u}(\alpha)+(C G(0)+D)^{T} \bar{L}_{y y}(\alpha)(C G(0)+D)\right] \nu_{0}\right\} \geq 0, \\
& \nu_{0} \in D^{+}
\end{aligned}
$$

Remark 3.1: The succinct form of Corollary 3.2 first appeared 
in [37] and [39] for abstract optimization problems. A similar result for a general optimal control problem was developed in [44].

Remark 3.2: The following specialization of OSS, considered in [24] and [25], occurs frequently in examples and simplifies Theorem 3.1 and Corollary 3.1. If $l=1+j+k$ and $g(y)=y$, then $M=I, \mu=\alpha$ and Theorem 3.1 can be modified by defining

$$
L(x, u, \alpha, \lambda)=\alpha^{T} \tilde{f}(x, u)+\lambda^{T} f(x, u)
$$

and omitting (3.5). Also, $\bar{L}_{y y}(\alpha)=0$ simplifies (3.8).

We now discuss several technical aspects of Theorem 3.1. The first-order necessary conditions are identical to [26, Theorem 3.1] except that there $U_{c}$ is assumed to be open, and hence (3.7) is replaced by

$$
\bar{L}_{u}(\alpha, \lambda)=\mathbf{0} .
$$

In both [26, Theorem 3.1] and the present paper no assertion is made as to whether $(\alpha, \lambda)$ is unique or $\alpha_{0}>0$. However, the second-order necessary conditions of our Theorem 3.1 are more general than [26, Theorem 3.2] since an auxiliary normality assumption or constraint qualification does not appear. Since it is not known in advance if there exists a multiplier $(\alpha, \lambda)$, in $\mathfrak{T}$ satisfying (3.8) for all $\left(\zeta_{0}, \nu_{0}\right) \in \mathfrak{D}$, the multiplier satisfying (3.8) may depend on the critical pair $\left(\zeta_{0}, \nu_{0}\right)$. A sufficient condition for the existence of $(\alpha, \lambda)$ satisfying (3.8) for all $\left(\zeta_{0}, \nu_{0}\right) \in \mathbb{D}$ is that $\mathfrak{N}$ consist of a unique element. To this end we have the following normality result which is analogous to [44, Theorem 3.5]. Let $M$ denote $M$ with its first row deleted.

Proposition 3.1: If

$$
\left[\begin{array}{c}
\hat{M} C \\
A
\end{array}\right] \mathbb{R}^{n}+\left[\begin{array}{c}
\hat{M} D \\
B
\end{array}\right] \text { cone }\left(U_{c}-\bar{u}\right)=\mathbb{R}^{j+k+n}
$$

then $\alpha_{0}>0$ for each $(\alpha, \lambda) \in \mathfrak{T}$. Furthermore, if $(\alpha, \lambda) \in \mathfrak{T}$ and

$$
\left[\begin{array}{c}
\hat{M} C \\
A
\end{array}\right] \hat{\beta}^{n}+\left[\begin{array}{c}
\hat{M} D \\
B
\end{array}\right] \text { cone } V=\mathbb{R}^{j+k+n},
$$

where

$$
V \triangleq\left\{\nu \in U_{c}-\bar{u}: \bar{L}_{u}(\alpha, \lambda) \nu=0\right\},
$$

then $\alpha_{0}>0$ and $(\alpha, \lambda)$ is the unique element of $\Re$.

Specializing to the case $\bar{u} \in$ int $U_{c}$, the normality condition (3.10) of [26] is obtained.

Corollary 3.4: Suppose $\bar{u} \in$ int $U_{c}$. If

$$
\operatorname{rank}\left[\begin{array}{cc}
\hat{M} C & \hat{M D} \\
A & B
\end{array}\right]=j+k+n
$$

then $\mathfrak{M}$ consists of a unique element $(\alpha, \lambda)$ and $\alpha_{0}>0$.

\section{Necessary Conditions for Steady-STate Optimality IN OPC}

In this section we present Theorem 4.1, first- and second-order necessary conditions for a local steady-state minimum of OPC $(\tau)$. The result follows from second-order weak-variation necessary conditions for a general optimal control problem given in Appendix B. The proof of Theorem 4.1 appears in Appendix C.

An interesting and useful feature of the necessary conditions is that if $\tau$ is excluded from a set of "harmonic" periods, then the first-order necessary conditions for a steady-state solution of OPC $(\tau)$ coincide with the (generally simpler) first-order necessary conditions for a local minimum of OSS. This restriction on $\tau$ is also largely responsible for the particularly simple form of the $\Pi$ test. These points will be discussed at the end of this section.
The set of "nonharmonic" periods is given by

$$
J \triangleq\left\{\tau: \tau \neq i \frac{2 \pi}{\omega}, \quad i \in \Theta, \omega \in \Omega\right\} \text {, }
$$

where

$$
\Omega \triangleq\{\omega>0: j \omega \text { is an eigenvalue of } A\},
$$

and, for $\tau \in J$, the set of "dynamic" critical directions is

$$
\begin{aligned}
D_{\tau} \triangleq & \left\{\left(\zeta_{0}, v\right) \in \mathbb{R}^{n} \times L_{\infty}\left([0, \tau], U_{c}-\bar{u}\right):\right. \\
& \left.\left(\zeta_{0}, \nu_{0}\right) \in \mathcal{D}, \nu_{0} \triangleq \frac{1}{\tau} \int_{0}^{\tau} v(t) d t\right\} .
\end{aligned}
$$

The Fourier coefficients $\nu_{i}$ of $v$ [see (A.8)] will be needed in the theorem statements. When $\tau \in \mathfrak{J}$ the second part of Theorem A.1 shows that the pair $\left(\zeta_{0}, v\right)$ can be thought of as determining a unique solution $z$ of (A.5) and (A.6).

For $\omega \geq 0$ such that $G(\omega)$ exists and for $(\alpha, \lambda) \in \mathscr{K}$, define the $\Pi$ matrix $\Pi(\omega, \alpha, \lambda) \in \widehat{B}^{m \times m}$ by

$$
\begin{aligned}
\Pi(\omega, \alpha, \lambda) \triangleq & G(\omega)^{*} \bar{L}_{x x}(\alpha, \lambda) G(\omega)+G(\omega)^{*} \bar{L}_{x u}(\alpha, \lambda) \\
& +\bar{L}_{u x}(\alpha, \lambda) G(\omega)+\bar{L}_{u u}(\alpha, \lambda) .
\end{aligned}
$$

When $\operatorname{det} A \neq 0$ denote $\Pi(\omega, \alpha, \lambda)$ by $\Pi(\omega, \alpha)$.

Theorem 4.1: If $(\bar{x}, \bar{u})$ is a local steady-state minimum of OPC $(\tau)$, where $\tau \in \mathcal{J}$, then $\mathfrak{N} \neq \emptyset$ and

$$
\begin{aligned}
& \max _{(\alpha, \lambda) \in \mathscr{M}}\left\{\zeta_{0}^{T} \bar{L}_{x x}(\alpha, \lambda) \zeta_{0}+2 \zeta_{0}^{T} \bar{L}_{x u}(\alpha, \lambda) \nu_{0}+\nu_{0}^{T} \bar{L}_{u u}(\alpha, \lambda) \nu_{0}\right. \\
& \quad+\left(C \zeta_{0}+D \nu_{0}\right)^{T} \bar{L}_{y y}(\alpha)\left(C \zeta_{0}+D \nu_{0}\right) \\
& \left.\quad+2 \sum_{i=1}^{\infty} \nu_{i}^{*} \Pi\left(i \frac{2 \pi}{\tau}, \alpha, \lambda\right) \nu_{i}\right\} \geq 0, \quad\left(\zeta_{0}, \nu\right) \in \mathbb{D}_{\tau} .
\end{aligned}
$$

If $\tau \in J$ and $\operatorname{det} A \neq 0$, then by Theorem A.1 the solution $z$ of (A.5) and (A.6) is uniquely determined by the control variation $v$. Thus, the set of critical directions is given by

$$
\mathbb{D}_{\tau}^{+}=\left\{v \in L_{\infty}\left([0, \tau], U_{c}-\bar{u}\right): \nu_{0} \triangleq \frac{1}{\tau} \int_{0}^{\tau} v(t) d t \in D^{+}\right\}
$$

and Theorem 4.1 specializes to the following result.

Corollary 4.1: If $(\bar{x}, \bar{u})$ is a local steady-state minimum of OPC ( $\tau$ ), where $\tau \in \mathcal{J}$, and $\operatorname{det} A \neq 0$, then $M^{-} \neq \phi$ and

$$
\begin{aligned}
& \max _{\alpha \in \mathscr{N}^{+}}\left\{\nu_{0}^{T}\left[\Pi(0, \alpha)+(C G(0)+D)^{\zeta} \bar{L}_{y y}(\alpha)(C G(0)+D)\right] \nu_{0}\right. \\
& \left.+2 \sum_{i=1}^{\infty} \nu_{i}^{*} \Pi\left(i \frac{2 \pi}{\tau}, \alpha\right) \nu_{i}\right\} \geq 0, \quad v \in \mathbb{D}_{\tau}^{+} .
\end{aligned}
$$

Note that the steady-state part of (4.1) coincides with the expression in (3.8). Also for the case $\operatorname{det} A \neq 0$ note that (3.15) can be written as

$$
\begin{aligned}
& \max _{\alpha \in \mathscr{N}^{+}}\left\{\nu_{0}^{T}\left[\Pi(0, \alpha)+(C G(0)+D)^{T} \bar{L}_{y y}(\alpha)(C G(0)+D)\right] \nu_{0}\right\} \geq 0, \\
& \nu_{0} \in \mathbb{D}^{+},
\end{aligned}
$$

which corresponds to the steady-state terms in (4.2).

\footnotetext{
${ }^{3}$ Note that there is a slight redundancy in this definition since $v \in L_{\infty}([0, \tau]$, $U_{c}-\bar{u}$ ) implies that $\nu_{0} \in U_{c}-\bar{u}$. This follows from a suitably modified version of [46, Theorem 1.6.13, p. 145].
} 
To help motivate the assumption $\tau \in \mathfrak{J}$ in Theorem 4.1 we now present the first-order necessary conditions for a local steady-state minimum of $\operatorname{OPC}(\tau)$ in the absence of this assumption. This result follows from the proof of Theorem 4.1 (see Appendix C).

Proposition 4.1: If $(\bar{x}, \bar{u})$ is a local steady-state minimum of $\operatorname{OPC}(\tau)$, then there exists nonzero $(\alpha, \lambda)$ satisfying (3.4), (3.5),

$$
\begin{gathered}
\bar{L}_{x}(\alpha, \lambda) \int_{0}^{\tau} e^{A t} d t=0, \\
{\left[\bar{L}_{u}(\alpha, \lambda)-\bar{L}_{x}(\alpha, \lambda) \int_{0}^{t} e^{-A \sigma} d \sigma B\right] \nu \geq 0,} \\
\nu \in U_{c}-\bar{u}, \text { a.a } t \in[0, \tau] .
\end{gathered}
$$

Clearly, when $\tau \in J(4.4)$ and (4.5) coincide with (3.6) and (3.7) since det $\int_{0}^{T} e^{A t} d t \neq 0$ (see Lemma A.1) implies $\bar{L}_{x}(\alpha, \lambda)=$ 0 . Another way of seeing this is to note that (4.5) is equivalent to (see Appendix C)

$$
\vec{L}_{u}(\alpha, p(t)) \nu \geq 0, \nu \in U_{c}-\bar{u}, \text { a.a. } t \in[0, \tau],
$$

where $p(t)$ is an adjoint state. As shown in Appendix C, the assumption $\tau \in \mathfrak{I}$ implies $p(t) \equiv \lambda$.

When $\tau \notin J$, det $\int_{0}^{\tau} e^{A t} d t=0$ and (4.4) may be satisfied even if $\bar{L}_{x}(\alpha, \lambda) \neq 0$. Hence, the set of $(\alpha, \lambda)$ satisfying (3.4), (3.5), (4.4), and (4.5) may be larger than the set of $(\alpha, \lambda)$ satisfying (3.4)-(3.7). The second-order necessary conditions for the case $\tau$ $\notin \mathfrak{J}$ (which are not stated here) are thus complicated in two ways: the possibly larger set of "dynamic" multipliers $(\alpha, \lambda)$ is more difficult to characterize than the "steady-state" multipliers because of the time dependency in (4.5), and the simple form of (4.1) is lost because $p(t)$ is not constant.

\section{The Generalized $\Pi$ Test}

In this section we obtain a second-order sufficient condition for locally proper from the second-order necessary conditions for a local steady-state minimum of OPC. The generalized $\Pi$ test, Theorem 5.1, is simply the converse of Theorem 4.1 specialized to the case most useful in practice in which the control variation consists of a steady-state term and a single sinusoidal term. We also consider the limiting cases of high and low frequency to obtain simplified tests for proper.

The following results involve the set of admissible fundamental frequencies

$$
W \triangleq\{\omega>0: i \omega \notin \Omega, i \in \mathbb{W}\}
$$

which corresponds to the set $\mathcal{I}$ of admissible periods. Note that $\omega$ $\in W$ if and only if $2 \pi / \omega \in \mathcal{J}$.

Theorem 5.1: Suppose $(\bar{x}, \bar{u})$ is a local minimum of OSS. If there exist $\omega \in \mathscr{W},\left(\zeta_{0}, \nu_{0}\right) \in \mathcal{D}$ and $\nu_{1} \in \mathrm{C}^{m}$ satisfying

$$
\bar{u}+\nu_{0}+\operatorname{Re} \nu_{1} e^{j t} \in U_{c}, \quad t \in[0,2 \pi],
$$

and

$$
\begin{aligned}
& \max _{(\alpha, \lambda) \in \mathfrak{K}}\left\{\zeta_{0}^{T} \bar{L}_{x x}(\alpha, \lambda) \zeta_{0}+2 \zeta_{0}^{T} \bar{L}_{x u}(\alpha, \lambda) \nu_{0}+\nu_{0}^{T} \bar{L}_{u u}(\alpha, \lambda) \nu_{0}\right. \\
& \left.\quad+\left(C \zeta_{0}+D \nu_{0}\right)^{T} \bar{L}_{y y}(\alpha)\left(C \zeta_{0}+D \nu_{0}\right)+1 / 2 \nu_{1}^{*} \Pi(\omega, \alpha, \lambda) \nu_{1}\right\}<0
\end{aligned}
$$

then $\mathrm{OPC}(2 \pi / \omega)$ is locally proper at $(\bar{x}, \bar{u})$.

For the case det $A \neq 0$ we have the following result.

Corollary 5.1: Suppose $(\bar{x}, \bar{u})$ is a local minimum of OSS and det $A \neq 0$. If there exist $\omega \in W, \nu_{0} \in D^{+}$, and $\nu_{1} \in \mathbb{G}^{m}$

\footnotetext{
${ }^{4}$ In accordance with ${ }^{3}(5.1)$ implies $\nu_{0} \in U_{c}-\bar{u}$.
}

satisfying (5.1) and

$$
\begin{aligned}
& \max _{\alpha \in M^{+}}\left\{\nu_{0}^{T}\left[\Pi(0, \alpha)+(C G(0)+D)^{T} \bar{L}_{y y}(\alpha)(C G(0)+D)\right] \nu_{0}\right. \\
&\left.+1 / 2 \nu_{1}^{*} \Pi(\omega, \alpha) \nu_{1}\right\}<0
\end{aligned}
$$

then $\mathrm{OPC}(2 \pi / \omega)$ is locally proper at $(\bar{x}, \bar{u})$.

Theorem 5.1 generalizes previous results from the literature in two important respects. As discussed in Section III, the "max" function in (5.1) reflects the absence of a normality condition. If a normality condition is satisfied (see Section III) then $\mathfrak{K}$ has a unique element, and hence "max" can be omitted. This is the case considered in [25] and [26]. The second feature of Theorem 5.1 is the presence of the steady-state terms in (5.1) involving $\zeta_{0}$ and $\nu_{0}$ which allow the $\Pi$ test to be used when $\bar{u}$ is an element of the boundary of $U_{c}$. In previous results [23]-[26] $\bar{u}$ is in the interior of $U_{c}$, and hence arbitrary weak variations in $U_{c}$ are permitted. In the present case the steady-state control component $\nu_{0}$ may be required so that the "perturbed" control remains inside $U_{c}$. This point is illustrated by an example in the next section. It is important to note that because of (3.13) the steady-state terms render (5.2) less likely to be satisfied. This is to be expected since $(\bar{x}, \bar{u})$ is assumed to be optimal over steady-state controls. Hence, loosely speaking, the generalized $\Pi$ test for locally proper is satisfied when the dynamic control variation has a greater effect on improving system performance than the steady-state control variation has on degrading it.

With regard to satisfying the control constraint (5.1), first note that $\nu_{1}$ in (5.1) corresponds to $2 \nu_{1}$ in (4.1). This substitution eliminates the need to carry along a factor of 2 in the succeeding development. If $\nu_{1}$ is real, then it can be seen that the control $\bar{u}+$ $\nu_{0}+\operatorname{Re} \nu_{1} e^{j t}=\bar{u}+\nu_{0}+\nu_{1} \cos t$ lies on the line segment connecting $\bar{u}+\nu_{0}-\nu_{1}$ to $\bar{u}+\nu_{0}+\nu_{1}$ and thus, since $U_{c}$ is convex, (5.1) is equivalent to

$$
\left\{\bar{u}+\nu_{0}-\nu_{1}, \bar{u}+\nu_{0}+\nu_{1}\right\} \subset U_{c} .
$$

If $\nu_{1}$ is purely imaginary, then (5.1) is equivalent to (5.4) with $\nu_{1}$ replaced by $\operatorname{Im} \nu_{1}$. In the general case $\nu_{1} \in \mathbb{C}^{m}, \operatorname{Re} \nu_{1}$ and $\operatorname{Im} \nu_{1}$ linearly independent, then the control $\bar{u}+\nu_{0}+\operatorname{Re} \nu_{1} e^{j t}$ traces out a two-dimensional ellipse in the affine subspace $\bar{u}+\nu_{0}+$ $\operatorname{span}\left\{\operatorname{Re} \nu_{1}, \operatorname{Im} \nu_{1}\right\}$. Thus, in general, it is not possible to replace (5.2) with a finite condition such as (5.4).

We now generalize observations made in [24] and [26] concerning the limiting cases of high and low frequency to deduce simplified tests for locally proper. Since $G(\omega) \rightarrow 0$ as $\omega \rightarrow \infty$ and $L$ is linear in $(\alpha, \lambda)$, it follows from elementary limit and continuity arguments that

$$
\lim _{\omega \rightarrow \infty} \Pi(\omega, \alpha, \lambda)=\bar{L}_{u u}(\alpha, \lambda)
$$

uniformly for $(\alpha, \lambda) \in \mathfrak{M}$. The following result is a corollary of Theorem 5.1.

Corollary 5.2: Suppose $(\bar{x}, \bar{u})$ is a local minimum of OSS. If there exist $\left(\zeta_{0}, \nu_{0}\right) \in \mathscr{D}$ and $\nu_{1} \in \mathcal{S}^{m}$ satisfying (5.1) and

$$
\begin{aligned}
& \max _{(\alpha, \lambda) \in \mathfrak{M}}\left\{\zeta_{0}^{T} \bar{L}_{x x}(\alpha, \lambda) \zeta_{0}+2 \zeta_{0}^{T} \bar{L}_{x u}(\alpha, \lambda) \nu_{0}+\nu_{0}^{T} \bar{L}_{u u}(\alpha, \lambda) \nu_{0}\right. \\
& \left.\quad+\left(C \zeta_{0}+D \nu_{0}\right)^{r} \bar{L}_{y y}(\alpha)\left(C \zeta_{0}+D \nu_{0}\right)+1 / 2 \nu_{1}^{*} \bar{L}_{u u}(\alpha, \lambda) \nu_{1}\right\}<0
\end{aligned}
$$

then there exists $\bar{\omega}>0$ such that $\operatorname{OPC}(2 \pi / \omega)$ is locally proper at $(\bar{x}, \bar{u})$ for all $\omega>\bar{\omega}$.

For the low-frequency limiting case we assume $\operatorname{det} A \neq 0$ so that

$$
\lim _{\omega \rightarrow 0} \Pi(\omega, \alpha)=\Pi(0, \alpha)
$$

uniformly for $\alpha \in \mathfrak{M}^{+}$. 
Corollary 5.4: Suppose $(\bar{x}, \bar{u})$ is a local minimum of OSS and $\operatorname{det} A \neq 0$. If there exist $\nu_{0} \in \mathbb{D}^{+}$and $\nu_{1} \in \mathbb{C}^{m}$ satisfying (5.1) and

$$
\begin{aligned}
& \max _{\alpha \in \Re^{+}}\left\{v_{0}^{T}\left[\Pi(0, \alpha)+(C G(0)+D)^{T} \bar{L}_{y y}(\alpha)(C G(0)+D)\right] \nu_{0}\right. \\
& \left.\quad+1 / 2 \nu_{1}^{*} \Pi(0, \alpha) \nu_{1}\right\}<0
\end{aligned}
$$

then there exists $\bar{\omega}>0$ such that $\operatorname{OPC}(2 \pi / \omega)$ is locally proper at $(\bar{x}, \bar{u})$ for all $\omega \in(0, \bar{\omega})$.

\section{EXAMPLES}

In this section we present examples to illustrate certain features of the results developed thus far. For each example it is assumed that $X=\mathbb{R}^{n}, U=\mathbb{R}^{m}, Y=\Re^{i}$ and the components of $f, \tilde{f}, x, u$, and $y$ are given by $f_{i}, \breve{f}_{i}, \cdots$, etc.

The first example, given in [26] to show that the results of [25] may fail in the absence of a normality condition, is now treated with the results of Sections III and IV. Let $n=m=k=1, j=0$,

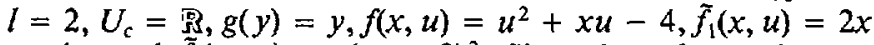
$+4 u$, and $\tilde{f}_{2}(x, u)=(u-2)^{2}$. Since the only steady-state admissible pair is $(\bar{x}, \bar{u})=(0,2)$, it is a local minimum of OSS. Using Remark 3.2 and det $A \neq 0$, it follows that $D^{+}=R, D_{\tau}^{+}$ $=L_{\infty}([0, \tau]$, 是 $)$, and

$$
\Pi(\omega, \alpha)=2\left[\frac{4-\omega^{2}}{4+\omega^{2}} \alpha_{0}+\alpha_{h}\right], \quad \omega \geq 0 .
$$

Since $\mathfrak{M}^{+}=\left\{\left(\alpha_{0}, \alpha_{h}\right\}: \alpha_{0}+\alpha_{h}=1\right\}$, we have

$$
\max _{\alpha \in \mathfrak{M}^{+}}\{\Pi(\omega, \alpha)\}=\Pi(\omega,(0,1))=2, \quad \omega \geq 0,
$$

which shows that (4.2) and (4.3) are satisfied.

The next example shows that in the absence of a normality condition the multiplier satisfying (3.8) may depend on the critical direction. This example is based upon [40, Example 2.1] which illustrates this phenomenon for nonlinear programming. Let $n=$ $j=2, l=m=3, k=0, U_{c}=\mathbb{R}^{3}, g(y)=y_{2} f_{1}(x, u)=u_{2}$, $f_{2}(x, u)=u_{3}, \tilde{f}_{1}(x, u)=2 x_{1} x_{2}+1 / 2 u_{1}^{2}-u_{2}^{2}, \tilde{f}_{2}(x, u)=2 x_{1} u_{1}$ $+1 / 2 x_{2}^{2}-1 / 2 u_{2}^{2}$ and $\tilde{f}_{3}(x, u)=2 x_{2} u_{1}+1 / 2 x_{1}^{2}-1 / 2 u_{3}^{2}$. That $(\bar{x}, \bar{u})=(0,0)$ is a local minimum of OSS can be seen in the following way. Of course, $\bar{u}_{2}=\bar{u}_{3}=0$. If one of the variables $x_{1}$, $x_{2}$, or $u_{1}$ is zero then the others must also be zero in order to satisfy (2.2) and not exceed the performance value of zero. Hence, the only remaining possibility is $x_{1}, x_{2}$, and $u_{1}$ all nonzero. However, this requires $x_{1} x_{2}, x_{1} u_{1}$, and $x_{2} u_{1}$ to be negative which is a contradiction since $\left(x_{1} x_{2}\right)\left(x_{1} u_{1}\right)\left(x_{2} u_{1}\right)=\left(x_{1} x_{2} u_{1}\right)^{2}>0$. Applying Theorem 3.1 we find that $\mathscr{M}=\left\{(\alpha, \lambda): \lambda=0, \alpha_{0}, \alpha_{1}\right.$, $\left.\alpha_{2} \geq 0, \alpha_{0}+\alpha_{1}+\alpha_{2}=1\right\}$ and $D=\left\{\left(\zeta_{0}, \nu_{0}\right) \in \mathbb{R}^{2} \times R^{3}: \zeta_{0}=\right.$ $\left.\left(\zeta_{01}, \zeta_{02}\right), \nu_{0}=\left(\nu_{01}, \nu_{02}, \nu_{03}\right), \nu_{02}=\nu_{03}=0\right\}$. Then for $\left(\zeta_{0}, \nu_{0}\right) \in$ $\mathscr{D}(3.8)$ has the form

$$
\left(\zeta_{0}^{T}, \nu_{0}^{T}\right)\left[\begin{array}{ccccr}
\alpha_{2} & 2 \alpha_{0} & 2 \alpha_{1} & 0 & 0 \\
2 \alpha_{0} & \alpha_{1} & 2 \alpha_{2} & 0 & 0 \\
2 \alpha_{1} & 2 \alpha_{2} & \alpha_{0} & 0 & 0 \\
0 & 0 & 0 & -2 \alpha_{0}-\alpha_{1} & 0 \\
0 & 0 & 0 & 0 & -\alpha_{2}
\end{array}\right]\left(\begin{array}{l}
\zeta_{0} \\
\nu_{0}
\end{array}\right) \geq 0
$$

To show that $\alpha$ satisfying (6.1) depends on $\left(\zeta_{0}, \nu_{0}\right)$, suppose (6.1) holds for some $\alpha$ and all $\left(\zeta_{0}, \nu_{0}\right) \in \mathcal{D}$. As shown in [40], choosing $\left(\xi_{0}, \nu_{0}\right)$ successively to be $(0,1,-1,0,0)^{T},(1,0,-1,0,0)^{T}$, and $(-1,1,0,0,0)^{T}$ and adding the results from (6.1) it follows that $\alpha=0$, which is a contradiction.

To determine if OPC is locally proper we consider only dynamic variations since $U_{c}$ is open. Noting

$$
\Pi(\omega, \alpha, \lambda)=\left[\begin{array}{ccc}
\alpha_{0} & \frac{-2 \alpha_{1}}{\omega} j & \frac{-2 \alpha_{2}}{\omega} j \\
\frac{2 \alpha_{1}}{\omega} j & \frac{\alpha_{2}}{\omega^{2}}-2 \alpha_{0}-\alpha_{1} & \frac{2 \alpha_{0}}{\omega^{2}} \\
\frac{2 \alpha_{2}}{\omega} j & \frac{2 \alpha_{0}}{\omega^{2}} & \frac{\alpha_{1}}{\omega^{2}}-\alpha_{2}
\end{array}\right], \omega>0,
$$

and choosing $\nu_{1}=(1,1,1)^{T}$ so that

$$
\begin{aligned}
\max _{(\alpha, \lambda) \in \mathscr{M}}\left\{\nu_{1}^{T} \Pi(\omega, \alpha, \lambda) \nu_{1}\right\} & =\max _{(\alpha, \lambda) \in \mathscr{M}}\left\{\nu_{1}^{T}[\operatorname{Re} \Pi(\omega, \alpha, \lambda)] \nu_{1}\right\} \\
& =4 \omega^{-2}-1, \quad \omega>0,
\end{aligned}
$$

it follows from Theorem 5.1 that $\operatorname{OPC}(2 \pi / \omega)$ is locally proper for $\omega>2$. Note that this result agrees qualitatively with Corollary 5.2 since

$$
\tilde{L}_{u u}(\alpha, \lambda)=\lim _{\omega \rightarrow \infty} \Pi(\omega, \alpha, \lambda)=\left[\begin{array}{ccc}
\alpha_{0} & 0 & 0 \\
0 & -2 \alpha_{0}-\alpha_{1} & 0 \\
0 & 0 & -\alpha_{2}
\end{array}\right]
$$

and hence

$$
\nu_{1}^{T} \bar{L}_{u u}(\alpha, \lambda) \nu_{1}=-1, \quad(\alpha, \lambda) \in \mathfrak{K} .
$$

The next example illustrates the need for a constant component of the control variation when the optimal steady-state control lies on the boundary of the control constraint set. Let $n=m=2, j$ $=k=0, l=1, U_{c}=\left\{\left(u_{1}, u_{2}\right) \in \Omega^{2}: u_{2} \geq 0\right\}_{2} g(y)=y, f_{1}(x$, $u)=x_{2}, f_{2}(x, u)=-x_{1}-x_{2}+u_{1}+u_{2}$, and $f(x, u)=2 u_{1}^{2}+$ $1 / 2 u_{2}^{2}-3 / 2 x_{2}^{2}$. It is easily seen that $(\bar{x}, \bar{u})=(0,0)$ is a local minimum of OSS, $\operatorname{det} A \neq 0, \lambda=0, \Re^{+}=\{1\}, \mathbb{D}^{+}=U_{c}$ and

$$
\Pi(\omega, 1)=\left[\begin{array}{cc}
4-Q(\omega) & -Q(\omega) \\
-Q(\omega) & 1-Q(\omega)
\end{array}\right], \quad \omega>0,
$$

where

$$
Q(\omega) \triangleq \frac{3 \omega^{2}}{\omega^{4}-\omega^{2}+1}, \quad \omega \geq 0 .
$$

We can avoid a constant component of the control variation ( $\nu_{0}$ $=0$ ) by taking $\nu_{1}=(\gamma, 0)^{T}, \gamma \neq 0$, so that the control variation lies in the boundary of $U_{c}$. In this case

$$
\nu_{1}^{T} \Pi(\omega, 1) \nu_{1}=\gamma^{2}[4-Q(\omega)] \geq \gamma^{2}>0, \quad \omega>0,
$$

and, thus, (5.2) does not hold. Suppose now we choose $\nu_{0}=\nu_{1}=$ $(0, \gamma)^{T}$, where $\gamma>0$, so that $\nu_{0} \in D^{+}$and (5.4) holds. It follows that

$$
\nu_{0}^{T} \Pi(0,1) \nu_{0}+\nu_{1}^{T} \Pi(\omega, 1) \nu_{1}=\gamma^{2}[2-Q(\omega)], \quad \omega>0 .
$$

Setting $\omega=1$ leads to the conclusion that $\operatorname{OPC}(2 \pi)$ is locally proper. This example clearly illustrates the mechanism for proper discussed previously, i.e., the improvement due to the dynamic component of the control variation outweighs the degradation caused by the steady-state component.

\section{Vi. Concluding Remarks}

Since the $\Pi$ test is essentially a specialization of weak-variation second-order necessary conditions for a general optimal control problem, further tests for proper can be obtained by applying recently obtained results from the optimal control literature. In particular, second-order necessary conditions exploiting strong control variations were given in [30], [44] and second-order 
necessary conditions involving "hybrid" variations (weak and strong simultaneously) appeared in [31]. New tests for proper based on these results may be useful in analyzing "chattering" solutions which arise, for example, in aircraft cruise problems [6], [11]-[14], [49].

Sufficiency conditions for steady-state optimality in OPC were given in [26] but were not considered in this paper. Since the results in [26] assume an open control constraint set and an auxiliary normality condition, generalization in the spirit of Theorem 5.1 appears possible. However, this is not feasible at the present time since higher order sufficiency theory for optimal control is not so advanced as necessity theory. The results of [41], which apply to general optimization problems and appear to be the strongest obtained thus far, may be applicable with some modification.

Finally, it should be stressed that the $\Pi$ test, being an optimality criterion, does not take into account system properties such as stability which govern the feasibility of utilizing local oscillations to improve system performance. As the results of [5] indicate, implementation of periodic controls can lead to both complex and unexpected behavior.

\section{APPENDIX A}

This Appendix contains basic results concerning periodic solutions of the system $\dot{z}=A z+B v$. We begin with a pair of technical lemmas concerning the imaginary eigenvalues of $A \in$ $R^{n \times n}$.

Lemma A.1: The condition

$$
\operatorname{det} \int_{0}^{\tau} e^{A t} d t \neq 0
$$

holds if and only if

$$
\operatorname{det}\left(j i \frac{2 \pi}{\tau} I_{n}-A\right) \neq 0, \quad i \in \text { 泟. }
$$

Proof. The result is easily obtained by considering the Jordan canonical form for $A$.

Noting the identity

$$
e^{A \tau}-I_{n}=A \int_{0}^{\tau} e^{A t} d t
$$

leads us to the following result.

Lemma A.2: The condition

$$
\operatorname{det}\left(e^{A \tau}-I_{n}\right) \neq 0
$$

holds if and only if $\operatorname{det} A \neq 0$ and (A.1) [or equivalently (A.2)] is satisfied.

Now consider the system

$$
\begin{gathered}
\dot{z}(t)=A z(t)+B v(t), \text { a.a. } t \in[0, \tau], \\
z(0)=z(\tau),
\end{gathered}
$$

where $A \in \mathbb{R}^{n \times n}, B \in \mathbb{R}^{n \times m}, z \in A C\left([0, \tau], \mathbb{R}^{n}\right)$, and $v \in$ $L_{\mathrm{l}}\left([0, \tau], \mathbb{R}^{m}\right)$. It is well known (see, e.g., [47, p. 148] or [26, p. 680]) that when (A.4) holds, (A.5) and (A.6) have a unique solution for each $v \in L_{1}\left([0, \tau], \AA^{m}\right)$. However, when (A.4) does not hold, multiple solutions may exist for a given $v$. To characterize these solutions let $v \in L_{1}\left([0, \tau]\right.$, $\left.\mathbb{R}^{m}\right)$ have the Fourier expansion

$$
v(t) \sim \sum_{i=-\infty}^{\infty} \nu_{i} \exp \left(j i \frac{2 \pi}{\tau} t\right)
$$

where $\nu_{0} \in \mathbb{R}^{m}, \nu_{i} \in \mathbb{G}^{m}$ and $\nu_{-i}=\nu_{i}, i \in \mathbb{N}$, are given by

$$
\nu_{i} \triangleq \frac{1}{\tau} \int_{0}^{\tau} \nu(t) \exp \left(-j i \frac{2 \pi}{\tau} t\right) d t .
$$

Since $z$ is absolutely continuous it possesses a uniformly and everywhere-convergent Fourier series (see, e.g., [48, p. 142]) denoted by

$$
z(t)=\sum_{i=-\infty}^{\infty} \zeta_{i} \exp \left(j i \frac{2 \pi}{\tau} t\right), \quad t \in[0, \tau],
$$

where the coefficients are defined as in (A.8).

Theorem A.1: Let $v \in L_{1}\left([0, \tau], \mathbb{R}^{m}\right)$ have the Fourier expansion (A.7). If $z \in A C\left([0, \tau], R^{n}\right)$ satisfies (A.5) and (A.6), then the Fourier coefficients $\zeta_{0} \in \mathbb{R}^{n}$ and $\left\{\zeta_{i}\right\}_{i=1}^{\infty} \subset \mathbb{C}^{n}$ of $z$ satisfy

$$
\begin{aligned}
-A \zeta_{0} & =B \nu_{0}, \\
\left(j i \frac{2 \pi}{\tau} I_{n}-A\right) \zeta_{i} & =B \nu_{i}, \quad i \in \mathbb{N} .
\end{aligned}
$$

Conversely, if $\zeta_{0} \in \mathbb{R}^{n}$ and $\left\{\zeta_{i}\right\}_{i=1}^{\infty} \subset \mathbb{C}^{n}, i \in \mathbb{N}$, satisfy (A.10) and (A.11), then $\zeta_{0}, \zeta_{1}, \cdots$ are the Fourier coefficients of an absolutely continuous function $z:[0, \tau] \rightarrow \mathbb{R}^{n}$ which satisfies (A.5) and (A.6).

Proof: Since term by term integration of the Fourier series of an integrable function is permissible (see, e.g., [48, p. 142]), (A.5) and (A.6) yield

$$
0=\frac{1}{\tau} \int_{0}^{\tau}[A z(t)+B v(t)] d t=A \zeta_{0}+B \nu_{0}
$$

Multiplying (A.5) by $\exp (-j i 2 \pi t / \tau)$ where $i \in \mathbb{N}$, applying (1/ $\tau) \int_{0}^{T}$ and using integration by parts, it can be seen that (A.11) holds.

To prove the converse we first show that $\zeta_{0}, \zeta_{1}, \zeta_{2}, \cdots$ are square summable, and hence are the Fourier coefficients of a function in $L_{2}\left([0, \tau], \mathbb{R}^{n}\right)$. To see this, note that $\left(j i 2 \pi / \tau I_{n}-A\right)^{-1}$ exists for all but a finite subset $\hat{I} \subset$ 则, and hence that

$$
\sum_{i=-\infty}^{\infty}\left|\zeta_{i}\right|^{2} \leq \sum_{i \in g y / f}\left|\left(j i \frac{2 \pi}{\tau} I_{n}-A\right)^{-1}\right| 2\left|v_{i}\right|^{2}+\sum_{i \in \mathcal{C}}\left|\zeta_{i}\right|^{2}
$$

Since $\left|\nu_{i}\right| \rightarrow 0$ as $i \rightarrow \infty$ and $\left|\left(j i 2 \pi / \tau I_{n}-A\right)^{-1}\right| \leq K / i$ for some $K>0$ and all $i \in \mathbb{N} / \hat{L}$, the right-hand side of (A.13) is finite.

Let $z$ denote the function in $L_{2}\left([0, \tau], R^{n}\right)$ whose Fourier coefficients are $\zeta_{0}, \zeta_{1}, \cdots$. Since $z$ is integrable, $q:[0, \tau] \rightarrow R^{n}$ defined by

$$
q(t)=\int_{0}^{t}[A z(s)+B v(s)] d s
$$

is absolutely continuous. Term by term integration in (A.14) along with (A.10) and (A.11) implies that, for $t \in[0, \tau]$,

$$
q(t)=\sum_{i=-\infty}^{\infty} \zeta_{i}\left[\exp \left(j i \frac{2 \pi}{\tau} t\right)-1\right]
$$

where the convergence is everywhere since $q$ is absolutely continuous.

Now, let $t^{\prime} \in[0, \tau]$ be a point at which the Fourier series for $z$ converges $[49, \mathrm{p} .153]$. Then we compute

$$
z\left(t^{\prime}\right)-q\left(t^{\prime}\right)=\sum_{i=-\infty}^{\infty} \zeta_{i}
$$

which shows that this sum exists. Hence, from (A.15) we can write

$$
q(t)=\sum_{i=-\infty}^{\infty} \zeta_{i} \exp \left(j i \frac{2 \pi}{\tau} t\right)-\sum_{i=-\infty}^{\infty} \zeta_{i}, \text { a.a. } t \in[0, \tau]
$$


or, with $z(0)$ redefined if necessary, $z(t)-z(0)=q(t)$, a.a $t \in$ $[0, \tau]$. Now redefine $z$ on a set of measure zero so that $z(t)=q(t)$ $+z(0)$ for all $t \in[0, \tau]$. Hence, $z$ is absolutely continuous and, by (A.14), satisfies (A.5). Finally, since $q(\tau)=0,($ A.6) holds, as required.

\section{APPENDIX B}

This section presents first- and second-order necessary conditions for optimal control which, with minor changes, appear as [44, Theorem 3.1]. This result is used in Appendix C for the proof of Theorem 4.1.

Let $\hat{n}, \hat{m} \in \mathbb{N}, \hat{j}, \hat{k} \in\{0\} \cup \mathbb{N}, \hat{X} \subset \mathrm{R}^{\hat{n}}, \hat{B} \subset \mathbb{R}^{2 n}$ and $\hat{U} \subset$ $\mathbb{R}^{\hat{m}}$ be open, $\hat{T} \triangleq\left[t_{1}, t_{2}\right]$, where $-\infty<t_{1}<t_{2}<\infty, \hat{\mathfrak{C}} \triangleq\{x \in$ $\left.A C(\hat{T}, \hat{X}):\left(\hat{x}\left(t_{1}\right), \hat{x}\left(t_{2}\right)\right) \in \hat{B}\right\}, \hat{\mathcal{U}} \subset M(T, \hat{U}), f: \hat{T} \times \hat{X} \times \hat{U} \rightarrow$ $\mathbb{R}^{\hat{n}}, \phi_{i}: \hat{B} \rightarrow \mathrm{R}, i \in\{0, \cdots, \hat{j}\}$, and $\psi: \hat{B} \rightarrow \mathrm{in}^{\hat{k}}$ (omit if $\hat{k}=0$ ).

\section{Optimal Control Problem (OC)}

Find $(\hat{x}, \hat{u}) \in \hat{X} \times \hat{\mathcal{U}}$ which minimizes

$$
J_{O C}(\hat{x}, \hat{u}) \triangleq \phi_{0}\left(\hat{x}\left(t_{1}\right), \hat{x}\left(t_{2}\right)\right)
$$

subject to

$$
\begin{gathered}
\left.\phi_{i}\left(\hat{x}\left(t_{1}\right), \hat{x}\left(t_{2}\right)\right) \leq 0, i \in\{1, \cdots, \hat{j}\} \text { (omit if } \hat{j}=0\right), \\
\left.\psi\left(\hat{x}\left(t_{1}\right), \hat{x}\left(t_{2}\right)\right)=0 \text { (omit if } \hat{k}=0\right), \\
\dot{x}(t)=\hat{f}(t, \hat{x}(t), \hat{u}(t)), \text { a.a. } t \in \hat{T}
\end{gathered}
$$

The necessary conditions require the following assumptions. Define the set

$$
\hat{U}^{\prime} \triangleq\{\hat{u}(t): \hat{u} \in \hat{u}, t \in \hat{T}\} .
$$

Assumption B.1: For all $t \in \hat{T}, \hat{f}(t, \cdot, \cdot)$ is $C^{\mathrm{l}}$ on $\hat{X} \times \hat{U}$; for all $(\hat{x}, \hat{u}) \in \hat{X} \times \hat{U}^{\prime}$, the components of $\hat{f}(\cdot, \hat{x}, \hat{u}), \hat{f}_{\hat{x}}(\cdot, \hat{x}, \hat{u})$ and $\hat{f}_{\hat{u}}(\cdot, \hat{x}, \hat{u})$ are measurable on $\hat{T}$; there exists $\beta_{1}(\cdot) \in L_{1}(\hat{T}$, R) such that for all $(t, \hat{x}, \hat{u}(\cdot)) \in \hat{T} \times \hat{X} \times \hat{u},|\hat{f}(t, \hat{x}, \hat{u}(t))|+$ $\left|\hat{f}_{\hat{x}}(t, \hat{x}, \hat{u}(t))\right|+\left|\hat{f}_{\hat{u}}(t, \hat{x}, \hat{u}(t))\right| \leq \beta_{1}(t) ; \phi_{0}, \cdots, \phi_{j}$ and $\psi$ (omit if $\hat{k}=0$ ) are $C^{1}$ on $\hat{B}$.

Assumption B.2: For all $t \in \hat{T}, \hat{f}(t, \cdot, \cdot)$ is $C^{2}$ on $\hat{X} \times \hat{O}$; for all $(\hat{x}, \hat{u}) \in \hat{X} \times \hat{U}^{\prime}$ the components of $\hat{f}_{\hat{x} \hat{x}}(\cdot, \hat{x}, \hat{u}), \hat{f}_{\hat{x} \hat{u}}(\cdot, \hat{x} \hat{u})$, and $\hat{f}_{\hat{u} \hat{u}}(\cdot, \hat{x}, \hat{u})$ are measurable on $\hat{T}$, thus there exists $\beta_{2}(\cdot) \in L_{1}(\hat{T}$, R) such that for all $(t, \hat{x}, \hat{u}(\cdot)) \in \hat{T} \times \hat{X} \times \hat{\mathcal{u}},\left|\hat{f}_{\hat{x} \hat{u}}(t, \hat{x}, \hat{u}(t))\right|$ $+\left|\hat{f}_{\hat{x} \hat{u}}(t, \hat{x}, \hat{u})\right|+\left|\hat{f}_{\hat{u} \hat{u}}(t, \hat{x}, \hat{u})\right| \leq \beta_{2}(t) ; \phi_{0}, \cdots, \phi_{\hat{j}}$ and $\psi$ (omit if $k=0$ ) are $C^{2}$ on $\hat{B}$.

A super-bar notation denotes evaluation on a solution $(\overline{\hat{X}}(\cdot)$, $\overline{\hat{u}}(\cdot))$ of $O C$. We regard the elements of $\hat{B}$ as pairs $\left(\hat{x}_{1}, \hat{x}_{2}\right) \in \bar{B}^{n}$ $\times R^{\hat{n}}$, and hence the notation $\bar{\phi}_{i \hat{x}_{1}}, \bar{\phi}_{i \hat{x}_{2}}$, etc., is interpreted accordingly.

The set of control variations is any convex set $\tilde{u}$ satisfying

$$
0 \in \tilde{\mathrm{U}} \subset(\hat{\mathcal{U}}-\overline{\hat{u}}(\cdot)) \cap L_{\infty}\left(\hat{T}, \Re^{\hat{m}}\right),
$$

and the set of critical directions is

$$
\hat{\mathscr{D}} \triangleq\left\{(\hat{z}, \hat{v}) \in A C\left(\hat{T}, \mathbb{R}^{\hat{n}}\right) \times \tilde{u}:\right.
$$

$(\hat{z}, \hat{v})$ satisfies (B.6)-(B.8)\},

where

$$
\begin{gathered}
\bar{\phi}_{i \hat{x}_{1}} \hat{z}\left(t_{1}\right)+\bar{\phi}_{i \hat{x}_{2}} \hat{z}\left(t_{2}\right) \leq 0, \quad i \in \hat{\jmath}_{A 0}, \\
\bar{\psi}_{i \hat{x}_{1}} \hat{z}\left(t_{1}\right)+\bar{\psi}_{i \hat{\gamma}_{2}} \hat{z}\left(t_{2}\right)=0 \text { (omit if } \hat{k}=0 \text { ), } \\
\dot{z}(t)=\overline{\hat{f}}_{\hat{x}}(t) \hat{z}(t)+\overline{\hat{f}}_{\hat{u}}(t) \hat{v}(t), \text { a.a. } t \in \hat{T},
\end{gathered}
$$

and

$$
\begin{aligned}
\hat{\mathfrak{S}}_{A 0} & \stackrel{\Leftrightarrow}{\triangleq}\{0\}, \quad \hat{j}=0, \\
& \triangleq\{0\} \cup\left\{i \in\{1, \cdots, \hat{j}\}: \bar{\phi}_{i}=0\right\}, \quad \hat{j}>0 .
\end{aligned}
$$

Finally, define a Lagrangian function $\mathcal{L}: \hat{B} \times \hat{\beta}^{1+\hat{j+k}} \rightarrow$ R by

$$
\mathcal{L}\left(\hat{x}_{l}, \hat{x}_{2}, \hat{l}\right)=\sum_{i=1}^{j} \hat{l}_{i} \phi_{i}\left(\hat{x}_{1}, \hat{x}_{2}\right)+\hat{l}_{\psi}^{T} \psi\left(\hat{x}_{1}, \hat{x}_{2}\right),
$$

where

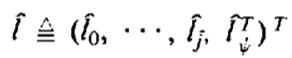

(omit $\psi$ and $\hat{l}$, when $\hat{k}=0$ ) and a Hamiltonian function $\mathfrak{H}: \hat{T} \times \hat{X}$ $\times \hat{U} \times \beta^{\hat{n}} \rightarrow \mathrm{\beta}$ by

$$
\mathcal{H C}(t, \hat{x}, \hat{u}, \hat{p}) \stackrel{\dot{\epsilon}}{=} \hat{p}^{T} \hat{f}(t, \hat{x}, \hat{u}) .
$$

Theorem $B .1$ : If $(\overline{\hat{x}}(\cdot), \overline{\hat{u}}(\cdot))$ solves $O C$ and Assumption B. 1 is satisfied, then there exist nonzero $\hat{l} \in \mathrm{R}^{1+\hat{j}+k}$ and $\hat{p} \in A C(\hat{T}$, $\mathrm{R}^{\hat{n}}$ ) such that

$$
\begin{gathered}
\hat{l}_{i} \geq 0, \quad i \in\{0, \cdots, \hat{j}\}, \\
\hat{l}_{i}=0, i \in\{0, \cdots, \hat{j}\} / \hat{\mathfrak{S}}_{A 0}, \\
\hat{p}\left(t_{1}\right)=-\overline{\mathfrak{L}}_{\hat{x}_{1}}\left(\hat{l}^{T}, \hat{p}\left(t_{2}\right)=\overline{\mathfrak{L}}_{\hat{x}_{2}}(\hat{l})^{T},\right. \\
\dot{\hat{p}}(t)=-\overline{\mathcal{K}}_{\hat{x}}(t, \hat{p}(t))^{T}, \text { a.a. } t \in \hat{T}, \\
\int_{t_{1}}^{t_{2}} \overline{\mathcal{K}}_{\hat{u}}(t, \hat{p}(t)) \tilde{v}(t) d t \geq 0, \tilde{v} \in \tilde{\mathcal{U}} .
\end{gathered}
$$

If, in addition, Assumption B. 2 is satisfied, then for each $(\hat{z}, \hat{v}) \in$ $\hat{D}$ there exist nonzero $\hat{l} \in \hat{\beta}^{i+\hat{j}+\hat{k}}$ and $\hat{p} \in A C\left(\hat{T}, \hat{R}^{\hat{n}}\right)$ such that (B.9)-(B.13) are satisfied and

$$
\begin{aligned}
& \hat{z}\left(t_{1}\right)^{T} \overline{\mathscr{L}}_{\hat{x}_{1} \hat{x}_{1}}(\hat{l}) \hat{z}\left(t_{1}\right)+2 \hat{z}\left(t_{1}\right)^{T} \overline{\mathscr{L}}_{\hat{x}_{1} \bar{x}_{2}}(\hat{l}) \hat{z}\left(t_{2}\right) \\
& \quad+\hat{z}\left(t_{2}\right)^{T} \overline{\mathfrak{L}}_{\hat{x}_{2} \hat{x}_{2}}(\hat{l}) \hat{z}\left(t_{2}\right)+\int_{t_{1}}^{t_{2}}\left[\hat{z}(t)^{T} \overline{\mathfrak{K}}_{\hat{x} \hat{x}}(t, \hat{p}(t)) \hat{z}(t)\right. \\
& \left.\quad+2 \hat{z}(t)^{T} \overline{\mathcal{K}}_{\hat{x} \hat{\imath} \hat{l}}(t, \hat{p}(t)) \hat{v}(t)+\hat{v}(t)^{T} \overline{\mathfrak{K}}_{\hat{u} \hat{u}}(t, \hat{p}(t)) \hat{v}(t)\right] d t \geq 0 .
\end{aligned}
$$

\section{APPENDiX C}

To prove Theorem 4.1, first note that the local optimality assumption can be treated by replacing $X$ and $U_{c}$ by $X \cap N_{\bar{X}}$ and $U_{c} \cap N_{\bar{t}}$, where $N_{x}$ and $N_{u}$ are sufficiently small neighborhoods of $\bar{x}$ and $\bar{u}$. Because the necessary conditions are homogeneous in state and control variations, $N_{\dot{x}}$ and $N_{u}$ play no role in the results. For convenience, we set $N_{\dot{x}}=\{\}^{n}$ and $N_{\bar{u}}=[\mathrm{f}\}^{m}$.

Let $\hat{n}=n+l, \hat{m}=m, \hat{j}=j, \hat{k}=k^{u}+n, \hat{T}=[0, \tau], \hat{X}=$ $X \times \widehat{\beta}^{i}, \hat{U}=U, \hat{\mathcal{U}}=L_{\infty}\left([0, \tau], U_{c}\right)$ and $\hat{B}=\left(\mathbb{R}^{n} \times N_{1}\right) \times$ ( $\mathrm{s}^{n} \times N_{2}$ ), where $N_{1}$ and $N_{2}$ are neighborhoods of $0 \in \mathbb{R}^{1}$ and $\tau \bar{y}$, respectively, sufficiently small so that $\tau^{-1}\left(N_{2}-N_{1}\right) \subset Y$. Also, let $\hat{x}=(x, \tilde{x}), \hat{u}=u, f(t, \hat{x}, \hat{u})=\left(f(x, u)^{T}, \tilde{f}(x, u)^{T}\right)^{T}$ and, for $\hat{x}_{1}=\left(x_{1}, \tilde{x}_{1}\right), \hat{x}_{2}=\left(x_{2}, \tilde{x}_{2}\right), \phi_{i}\left(\hat{x}_{1}, \hat{x}_{2}\right)=\tau g_{i}\left(\tau^{-1}\left(\tilde{x}_{2}-\right.\right.$ $\left.\left.\tilde{x}_{1}\right)\right)$ and $\psi\left(\hat{x}_{1}, \hat{x}_{2}\right)=\left(\tau h\left(\tau^{-1}\left(\tilde{x}_{2}-\tilde{x}_{1}\right)\right)^{T},\left(x_{2}-x_{1}\right)^{T}\right)^{T}$. It is easily seen that Assumption B.1 and B.2 are satisfied.

Letting $\hat{l}=\left(\alpha^{T}, \lambda^{T}\right)^{T}, \mathcal{L}\left(\hat{x}_{1}, \hat{x}_{2}, \hat{l}\right)=\alpha^{T} \tau g\left(\tau^{-1}\left(\tilde{x}_{2}-\tilde{x}_{1}\right)\right)+$ $\lambda^{T}\left(x_{2}-x_{1}\right), \hat{p}=(p, \tilde{p})$ and $\mathcal{H}(t, \hat{x}, \hat{u}, \hat{p})=p^{T} f(x, u)+$ $\tilde{p}^{T} \tilde{f}(x, u)$, we see that (B.9) is equivalent to (3.4) and (B.11) becomes $p(0)=p(\tau)=\lambda$ and $\tilde{p}(0)=\tilde{p}(\tau)=\left(\alpha^{T} M\right)^{T}$. From (B.12) it follows that $\tilde{p} \equiv\left(\alpha^{T} M\right)^{T}, \dot{p}(t)^{T}=-p(t)^{T} A-$ 
$\alpha^{T} M C$, and hence

$$
\begin{aligned}
p(t)^{T} & =\left[\lambda^{T}-\alpha^{T} M C \int_{0}^{t} e^{A \sigma} d \sigma\right] e^{-A t} \\
& =\lambda^{T}-\bar{L}_{x}(\alpha, \lambda) \int_{0}^{t} e^{A \sigma} d \sigma e^{-a t},
\end{aligned}
$$

where $\mu$ in $\bar{L}_{x}(\alpha, \lambda)$ is given by (3.5). From $p(\tau)=\lambda$ we obtain (4.4). Since $\tau \in J$, Lemma A. 1 implies det $\int_{0}^{\tau} e^{A t} d t \neq 0$, and hence (3.6) holds.

Next let $\tilde{\mathcal{U}}=L_{\infty}\left([0, \tau], U_{c}-\bar{u}\right)$ and note that by [44, Remark 3.6] (B.13) is equivalent to

$$
\overline{\mathfrak{H}}_{\hat{u}}(t, \hat{p}(t)) \tilde{v}(t) \geq 0, \tilde{v} \in \tilde{\mathcal{U}}, \text { a.a. } t \in[0, \tau] .
$$

Choosing $\tilde{v}(t) \equiv \nu \in U_{c}-\bar{u}$, it can be seen that (C.2) is equivalent to (4.6). Since (3.6) and (C.1) imply $p(t) \equiv \lambda,(3.7)$ follows from (4.6). Alternatively, (3.7) can be obtained by writing (4.6) in the equivalent form (4.5) and using (3.6).

For the second-order necessary conditions we see that $\hat{D}$ consists of pairs $(\hat{z}, v)$, where $\hat{z}=(z, \tilde{z}),(z, v)$ satisfies (A.5) and (A.6), $\dot{\tilde{z}}(t)=C z(t)+D v(t)$, a.a. $t \in[0, \tau]$, and $\left(\zeta_{0}, v\right) \in D_{\tau}$. Since $\tau \in J$, Lemma A.1, (3.3) and Theorem A.1 imply that the solution $z$ of (A.5) and (A.6) is uniquely determined by $\left(\zeta_{0}, v\right)$. Moreover, since (as will be seen) the only term involving $z$ needed in the evaluation of (B.14) is

$$
\tilde{z}(\tau)-\tilde{z}(0)=\tau\left(C \zeta_{0}+D \nu_{0}\right)
$$

$\hat{D}$ is completely characterized by $D_{r}$.

From (C.3), the first three terms of (B.14) become

$$
\tau\left(C \zeta_{0}+D \nu_{0}\right)^{r} \bar{L}_{y y}(\alpha)\left(C \zeta_{0}+D \nu_{0}\right) .
$$

Next, using $p(t) \equiv \lambda$ and $\tilde{p}=\left(\alpha^{T} M\right)^{T}$ the terms under the integral in (B.14) are

$$
\begin{aligned}
& z(t)^{T} \bar{L}_{x x}(\alpha, \lambda) z(t)+2 z(t)^{T} \bar{L}_{x u}(\alpha, \lambda) v(t) \\
& \quad+v(t)^{T} \bar{L}_{u u}(\alpha, \lambda) v(t)
\end{aligned}
$$

Using the Fourier expansions for $z$ and $v$, noting that, since $\tau \in$ J, (A.11) can be written as

$$
\zeta_{i}=G\left(i \frac{2 \pi}{\tau}\right) \nu_{i}, \quad i \in \mathrm{N},
$$

and integrating (C.S) term by term (this is permissible since $z$ and $v$ are square integrable), the integral in (B.14) becomes

$$
\begin{aligned}
& \tau\left[\zeta_{0}^{T} \bar{L}_{x x}(\alpha, \lambda) \zeta_{0}+2 \zeta_{0}^{T} \bar{L}_{x u}(\alpha, \lambda) \nu_{0}+\nu_{0}^{T} \bar{L}_{u u}(\alpha, \lambda) \nu_{0}\right. \\
& \left.+\sum_{\substack{i=-\infty \\
i \neq 0}}^{\infty} \nu_{i}^{*} \Pi\left(i \frac{2 \pi}{\tau}, \alpha, \lambda\right) \nu_{i}\right] .
\end{aligned}
$$

Finally, adding (C.4) and (C.7) and dividing by $\tau$ yields the expression in (4.1).

\section{ACKNOWLEDGMENT}

The author wishes to thank E. G. Gilbert for suggesting the topic of this paper and for numerous helpful discussions. Typing support was provided by Lincoln Laboratory, M.I.T.

\section{REFERENCES}

[1] J. M. Douglas, Process Dynamics and Control, Vol. II. Englewood Cliffs, NJ: Prentice-Hall, 1972.
[2] J. E. Bailey, "Periodic operation of chemical reactors: A review," Chem. Eng. Commun., vol. 1, pp. 111-124, 1973.

[3] E. J. Noldus, "Periodic optimization of a chemical reactor system using perturbation methods," J. Eng. Math., vol. II, pp. 49-66, 1977.

[4] M. Matsubara and K. Onogi, "'Stabilized suboptimal periodic control of a chemical reactor," IEEE Trans. Automat. Contr., vol. AC-23, pp. 1005-1008, 1978.

[5] D. Sincic and J. E. Bailey, "Pathological dynamic behavior of forced periodic chemical processes," Chem. Eng. Sci., vol. 32, pp. 281286.

[6] N. R. Zagalsky, R. E. Irons, and R. L. Schultz, "The energy state approximation and minimum-fuel fixed-range trajectory," J. Aircraft, vol. 8, pp. $488-490,1971$.

[7] R. L. Schultz and N. R. Zagalsky, "Aircraft performance optimization," J. Aircraft, vol. 9, pp. 108-114, 1972 .

[8] J. L. Speyer, "On the fuel optimality of cruise," J. Aircraft, vol. 10, pp. $763-765,1973$.

[9] R: Schultz, "Fuel optimality of cruise," J. Aircraft, vol. 11, pp. 586587, 1974.

[10] J. L. Speyer, "Nonoptimality of the steady-state cruise for aircraft," AIAA J, vol. 14, pp. 1604-1610, 1976.

[11] E. G. Gilbert and M. G. Parsons, "Periodic control and the optimality of aircraft cruise," J. Aircraft, vol. 13, pp. 828-830, 1976.

[12] E. G. Gilbert, "Vehicle cruise: Improved fuel economy by periodic control," Automatica, vol. 12, pp. 159-166, 1976.

[13] D. T. Lyons, "Improved aircraft cruise by periodic control," Ph.D. dissertation, Univ. Michigan, Ann Arbor, 1980.

[14] E. G. Gilbert and D. T. Lyons, "The improvement of aircraft specific range by periodic control," in Proc. AIAA Guid. Contr. Conf. Albuquerque, NM, 1981.

[15] J. C. Vrana, "Dynamic soaring," Trans. Can. Aerosp. Inst., vol. 2 pp. 94-106, 1968.

[16] F. Hendriks, "Dynamic soaring," Ph.D. dissertation., Univ. Calif., Los Angeles, 1972.

[17] P. Dorato and H. K. Knudsen, "Periodic optimization with applications to solar energy control," Automatica, vol. 15, pp. 673-676, 1979.

[18] J. Timonen and R. Hamalainen, "Optimal periodic control strategies in a dynamic pricing problem," Int. J. Syst. Sci., vol. 10, pp. 197-205, 1979.

[19] D. A. Spyker, "Application of aptimal control theory to cardio-circulatory assist devices," Ph.D. dissertation, Univ. Minnesota, 1969.

[20] J. E. Bailey and F. J. M. Horn, "Comparison between two sufficient conditions for improvement of an optimal steady-state process by periodic operation," J. Optimiz. Theory Appl., vol. 7, pp. 378-384, 1971.

[21] A. Marzollo, Ed., Periodic Optimization, Vols. 1, 2. New York: Springer-Verlag, 1972

[22] E. G. Gilbert, "Optimal periodic control: A general theory of necessary conditions," SIAM J. Contr. Optimiz., vol. 15, pp. 717 746. 1977.

[23] G. Guardabassi, "Optimal steady state versus periodic control: A circle criterion," Richerche Di Automatica, vol. 2, pp. 240-252, 1971.

[24] S. Bittanti, G. Fronza, and G. Guardabassi, "Periodic control: A frequency domain approach," IEEE Trans. Automat. Contr., vol. AC-18, pp. 33-38, 1973.

[25] W. L. Chan and S. K. Ng, "Normality and proper performance improvement in periodic control," J. Optimiz. Theory Appl., vol. 29 , pp. $215-229,1979$

[26] D. S. Bernstein and E. G. Gilbert, "Optimal periodic control: The $\pi$ test revisited," IEEE Trans. Automat. Contr., vol. AC-25, pp. 673$684,1980$.

[27] G. Guardabassi and N. Schiavoni, "Boundary optimal constant control versus periodic operation," in Proc. 6th Triennial World Congress, Int. Fed. Automat. Contr., Boston, MA, 1975.

[28] M. R. Hestenes, Calculus of Variations and Optimal Control Theory. New York: Wiley, 1964.

[29] J. Warga, "A second-order Lagrangian condition for restricted control problems," J. Optimiz. Theory Appl., vol. 24, pp. 475-483, 1978.

[30] , "A second-order condition that strengthens Pontryagin's maximum principle," J. Differential Equations, vol. 28, pp. 284-307, 1978.

[31] - "A hybrid relaxed-Lagrangian second-order condition for minimum," in Differential Games and Control Theory (Proc. 3rd Kingston Conf., Part A) New York: Marcel Dekker, 1979, pp. 7794.

[32] G. A. Bliss, "Normality and abnormality in the calculus of variations," Trans. Amer. Math. Soc., vol. 43, pp. 365-376, 1938.

[33] G. A. Bliss, Lectures on the Calculus of Variations. Chicago, IL: Univ. Chicago Press, 1946.

[34] A. Fiacco and G. McCormick, Nonlinear Programming. New York: Wiley, 1968.

[35] E. J. Messerli and E. Polak, "On second-order necessary conditions for optimality," SIAM J. Contr. Optimiz., vol.7, pp.272-291, 1969.

[36] C. Virsan, "Necessary conditions on extremality of high order," Revue Roumaine Mathemat. Pures Appl., vol. 28, pp. 591-611, 1973. 
[37] E. S. Levitin, A. A. Miljutin, and N. Osmoloyskii, "On conditions for local minimum in a problem with constraints" (in Russian) in Mathematical Economics and Functional Analysis, B. S. Mitjagin, Ed. Moscow: Nauka, 1974

[38] K. H. Hoffmann and H. J. Kornstaedt, "High-order necessary conditions in abstract mathematical programming," $J$. Optimiz. Theory Appl., vol. 26, pp. 533-568, 1978.

[39] A. D. Ioffe "Necessary and sufficient conditions for a local minimum 3: Second-order conditions and augmented duality," SIAM J. Contr. Optimiz., vol. 17, pp. 266-288, 1979.

[40] A. Ben-Tal, "Second-order and related extremality conditions in nonlinear programming," J. Optimiz. Theory Appl., vol. 31, pp. $143-165,1980$.

[41] F. Lempio and J. Zowe, "High-order optimality conditions," in Modern Applied Mathematics-Optimization and Operations Research, B. Korte, Ed. Amsterdam, The Netherlands: North-Holland, 1982 , pp. $147-193$.

[42] A. Linnemann, "Higher-order necessary conditions for infinite and semi-infinite optimization," J. Optimiz. Theory Appl., vol. 38, pp. 483-511, 1982.

[43] D. S. Bernstein, "A systematic approach to higher-order necessary conditions in optimization theory," SIAM J. Contr. Optimiz., vol, 22, pp. 211-238, 1984.

[44] E. G. Gilbert and D. S. Bernstein, "Second-order necessary conditions in optimal control: Accessory-problem results without normality," $J$. Optimiz. Theory Appl., vol. 41, pp. 75-106, 1983.

[45] E. J. McShane, "Sufficient conditions for a weak relative minimum in the problem of Bolza," Trans. Amer. Math. Soc., vol. 52, pp. 344$379,1942$.

[46] J. Warga, Optimal Control of Differential and Functional Equations. New York: Academic, 1972.
[47] J. K. Hale, Ordinary Differential Equations. New York: Wiley, 1969.

[48] C. S. Rees, S. M. Shah, and C. V. Stanojevic, Theory and Applications of Fourier Analysis. New York: Marcel Dekker, 1981.

[49] S. C. Houlihan, E. M. Cliff, and H. J. Kelley, "Study of chattering cruise," J. Aircraft, vol. 19, pp. 119-124, 1982.

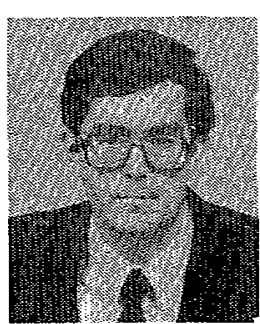

Dennis S. Bernstein (M'82) received the Sc.B. degree in applied mathematics from Brown University, Providence, RI, and the M.S.E. and $\mathrm{Ph} . \mathrm{D}$. degrees from the Computer, Information and Control Engineering Program at the University of Michigan, Ann Arbor.

After spending two years at Lincoln Laboratory, Massachusetts Institute of Technology, Lexington, he joined the Controls Analysis and Synthesis Group of the Government Aerospace Systems Division, Harris Corporation, Melbourne, FL. His current research interests include the optimal projection/maximum entropy approach to designing low-order robust controllers for high-order uncertain systems such as large flexible space structures modeled by stochastic partial differential equations.

Dr. Bernstein is a member of the Society for Industrial and Applied Mathematics and the American Institute of Aeronautics and Astronautics. 